\title{
Assessment of Japanese healthy children's nutritional status using Waterlow classification ${ }^{*}$
}

\author{
Yukie Higashiyama, Masaru Kubota\#, Satoko Oshima, Marie Mibu, Yuka Yasui, Ayako Nagai
}

Department of Human Life and Environment, Nara Women's University, Nara, Japan;

${ }^{\#}$ Corresponding Author: masaru kubota@chime.ocn.ne.jp

Received 14 September 2012; revised 17 October 2012; accepted 22 October 2012

\begin{abstract}
Malnutrition is associated with an increased risk of morbidity and mortality, and may case an undesirable effect on the growth and development in children. There have been several reports about the prevalence of malnutrition in hospitalized children, but reports about malnutrition in a large number of healthy children are quite limited. The aim of this study was to evaluate the prevalence of likelihood of malnutrition in Japanese healthy children. We retrospectively reviewed data of height and weight at a regular health check in 7517 healthy children (age 3 - 17; 3747 boys, 3770 girls) during 2008 and 2010 in Nara city, Japan. The data were evaluated using Waterlow classification, i.e., weight-for-height (W/H) and height-for-age (H/A). The prevalence of under-nutrition, as estimated by the values of W/H less than $90 \%$ and H/A less than $95 \%$, were $20.1 \%$ and $8.3 \%$, respectively, and this prevalence of malnutrition risk tended to vary substantially with age. The peak levels of the prevalence were found to be at around 12 years old for both sexes in $W / H$, and at around $\mathbf{1 1}$ years old for boys and at around 10 years old for girls in H/A. We have clearly demonstrated the existence of certain percentages of the likelihood of malnutrition at different ages in healthy children. These findings suggest that when we want to evaluate the nutritional status using Waterlow classification in hospitalized children, we should be careful by taking such reference values into account.
\end{abstract}

Keywords: Healthy Children; Malnutrition; Nutritional Assessment; Waterlow Classification

\section{INTRODUCTION}

Malnutrition is a state in which a deficiency or excess

"The authors declare that we have no conflicts of interest of any kind in regard to the work presented here. of nutrients may increase morbidity or mortality. Several investigators have reported on malnutrition and a risk of under-nutrition such as wasted or stunted condition or overweight in both hospitalized general patients [1-3] and those with specific disorders [4,5]. Since undernutrition and disease interact with each other adversely, adequate and prompt nutritional supports are required. There are several methods for evaluating a patient's nutritional status, including anthropometric measurements, dietary intake, biochemical parameters, and resting energy expenditure [6-8]. In hospitalized children, there is a good chance to use all of these measures; however, in healthy children, especially as a large cohort, it is difficult to perform these methods, except for anthropometric measures. Among several anthropometric measures, height-for-age (H/A) and weight-for-height $(\mathrm{W} / \mathrm{H})$ have been widely used in various clinical settings. $\mathrm{H} / \mathrm{A}$ and $\mathrm{W} / \mathrm{H}$ were first presented by Gomez et al. in 1956 [9] and by Waterlow in 1972 [10], respectively. Later, Waterlow put the two measures together and defined $\mathrm{H} / \mathrm{A}$ as an indicator for chronic nutritional status and $\mathrm{W} / \mathrm{H}$ as an indicator for acute nutritional status [11]. Therefore, both measures are often called "Waterlow classification" in the literature. Waterlow classification has been widely used in hospitalized children for evaluating nutritional status on admission and to track changes over time during hospitalization $[1,2,5,12]$. However, there are very few large-scale studies on the $\mathrm{H} / \mathrm{A}$ and $\mathrm{W} / \mathrm{H}$ scores in healthy children. The reference values are potentially important for adequate assessment of sick children in comparison with a healthy population. Therefore, in this study, we tried to determine the reference values and an incidence of malnutrition among healthy children at $3-17$ years of age in a large cohort of more than 7000 participants.

\section{MATERIAL AND METHODS}

\subsection{Participants}

To gain permission for the use of data obtained at an 
annual health check, we first sent a letter to 30 randomly selected principals of senior high schools, junior high schools, elementary schools, or kindergartens in Nara city between 2008 and 2010. Due to the restrictions on the use of personal information, we obtained consent from approximately $60 \%$ of the principals. We went to those schools to collect the data on a paper basis and in total enrolled 7517 healthy children (3747 boys and 3770 girls) aged 3 to 17 years old in this study (Table 1). Health checks were performed in April or May every year and were done by school teachers or school nurses who were well trained. Body weight was measured to the nearest $0.1 \mathrm{~kg}$, and height was measured to the nearest $0.1 \mathrm{~cm}$. At the same time, we recorded each subject's birth date to calculate respective standard weight and height.

\subsection{Waterlow Classification}

The Waterlow classification measures of $\mathrm{W} / \mathrm{H}$ and $\mathrm{H} / \mathrm{A}$ are calculated according to the following formula: $\mathrm{W} / \mathrm{H}=$ [observed weight/standard weight (for the same age and height and sex)] $\times 100$, and $\mathrm{H} / \mathrm{A}=$ [observed height/standard height (for same age and sex)] $\times 100$ [11] We used the official Japanese children's anthropometric data for children age 5 and over published by the Ministry of Education, Culture, Sports, Science and Technology [13], and for those age 4 and under published by the Ministry of Health, Labor and Welfare [14], as the standard weight and height. The criteria for the diagnosis of

Table 1. Number of participants

\begin{tabular}{|c|c|c|c|}
\hline Age (years) & Total (n) & Boys & Girls \\
\hline $3 \sim$ & 729 & 376 & 353 \\
\hline $4 \sim$ & 794 & 403 & 391 \\
\hline $5 \sim$ & 568 & 286 & 282 \\
\hline $6 \sim$ & 468 & 239 & 229 \\
\hline $7 \sim$ & 389 & 199 & 190 \\
\hline $8 \sim$ & 385 & 196 & 189 \\
\hline $9 \sim$ & 308 & 156 & 152 \\
\hline $10 \sim$ & 303 & 153 & 150 \\
\hline $11 \sim$ & 370 & 184 & 186 \\
\hline $12 \sim$ & 740 & 358 & 382 \\
\hline $13 \sim$ & 609 & 294 & 315 \\
\hline $14 \sim$ & 490 & 238 & 257 \\
\hline $15 \sim$ & 493 & 239 & 254 \\
\hline $16 \sim$ & 374 & 184 & 190 \\
\hline $17 \sim$ & 492 & 242 & 250 \\
\hline All ages & 7517 & 3747 & 3770 \\
\hline
\end{tabular}

malnutrition were for $\mathrm{W} / \mathrm{H},<70 \%$ severe, $70 \%-80 \%$ moderate, $81 \%$ - 90\% mild under-nutrition, and $\geq 110 \%$ overweight, and for $\mathrm{H} / \mathrm{A},<85 \%$ severe, $85 \%$ - $89 \%$ moderate, and 90\% - 95\% mild under-nutrition. Differences between the prevalence of malnutrition among different age groups were examined using a population proportion test (SPSS ver.19). $p$ values less than 0.05 were considered significant.

\section{RESULTS}

\subsection{Prevalence of Children at Risk for Malnutrition}

The prevalence of children at risk for malnutrition according to Waterlow classification is summarized in Table 2 . Among our study subjects, 1513 (20.1\%) and 624 $(8.3 \%)$ children were found to be at some grade of malnutrition based on the evaluation of $\mathrm{W} / \mathrm{H}$ and $\mathrm{H} / \mathrm{A}$, respectively. Of the former 1513 children, $10(0.1 \%), 177$ $(2.4 \%)$, and $1326(17.6 \%)$ children were at levels of severe, moderate, and mild under-nutrition, respectively. On the other hand, of the latter 624 children, none, 24 $(0.3 \%)$, and $600(8.0 \%)$ children were at levels of severe, moderate, and mild under-nutrition, respectively. Eight hundred and nine $(10.8 \%)$ children were assessed as being overweight.

\subsection{Change in the Prevalence of Children at Risk for Malnutrition with Age}

Figure 1 indicates the prevalence of children at risk for under-nutrition with age. The prevalence at any grades of under-nutrition by $\mathrm{W} / \mathrm{H}$ tended to reach their peaks at the age of 12 in both sexes. Actually, 121 (33.8\%) boys and 141 (36.9\%) girls were found to be under-nourished at 12 years old. When we compared the prevalence of under-nutrition at 12 years old with other ages, the differences were statistically significant. In the measure of $\mathrm{H} / \mathrm{A}$, the prevalence of under-nutrition was highest at the age of 11 years for boys $(30 ; 16.3 \%)$ and 10 years for girls $(25 ; 16.7 \%)$.

\section{DISCUSSION}

The assessment of nutritional status is pivotal in the pediatric field for dealing with both healthy and sick children. In studies of children on hospital wards, a varied number of patients was diagnosed to be malnourished depending upon the definition of malnutrition and the method of nutritional assessment [1-5]. In the present study, the definition of malnutrition in cluded both under-nutrition (underweight) and over-nutrition (overweight). Without the comprehension of healthy children's nutritional status for comparison, it is difficult to 
Table 2. Comparison of the distribution of participants as judged by $\mathrm{W} / \mathrm{H}$ and $\mathrm{H} / \mathrm{A}$.

\begin{tabular}{|c|c|c|c|c|c|c|c|}
\hline & & & $\mathrm{W} / \mathrm{H}^{*}$ & & & & \\
\hline & & Severe ${ }^{* *}$ & Moderate $^{* *}$ & $\operatorname{Mild}^{* *}$ & Overweight & Normal & Total \\
\hline \multirow[t]{5}{*}{$\mathrm{H} / \mathrm{A}^{*}$} & Severe & $0^{* * *}$ & 0 & 0 & 0 & 0 & 0 \\
\hline & Moderate & 0 & 1 & 1 & $5(0.1)$ & $17(0.2)$ & $24(0.3)$ \\
\hline & Mild & 1 & $18(0.2)$ & $105(1.4)$ & $72(1.0)$ & $404(5.4)$ & $600(8.0)$ \\
\hline & Normal & $9(0.1)$ & $158(2.1)$ & $1220(16.2)$ & 732 (9.7) & $4774(63.5)$ & $6893(91.7)$ \\
\hline & Total & $10(0.1)$ & $177(2.4)$ & $1326(17.6)$ & 809 (10.8) & $5195(69.1)$ & $7517(100)$ \\
\hline
\end{tabular}

"W/H and H/A indicate weight-for-height and height-for-age, respectively; ${ }^{* *}$ "Severe", "Moderate", and "Mild" indicate under-nutrition; ${ }^{* * *}$ Numbers in parentheses indicate percentages.

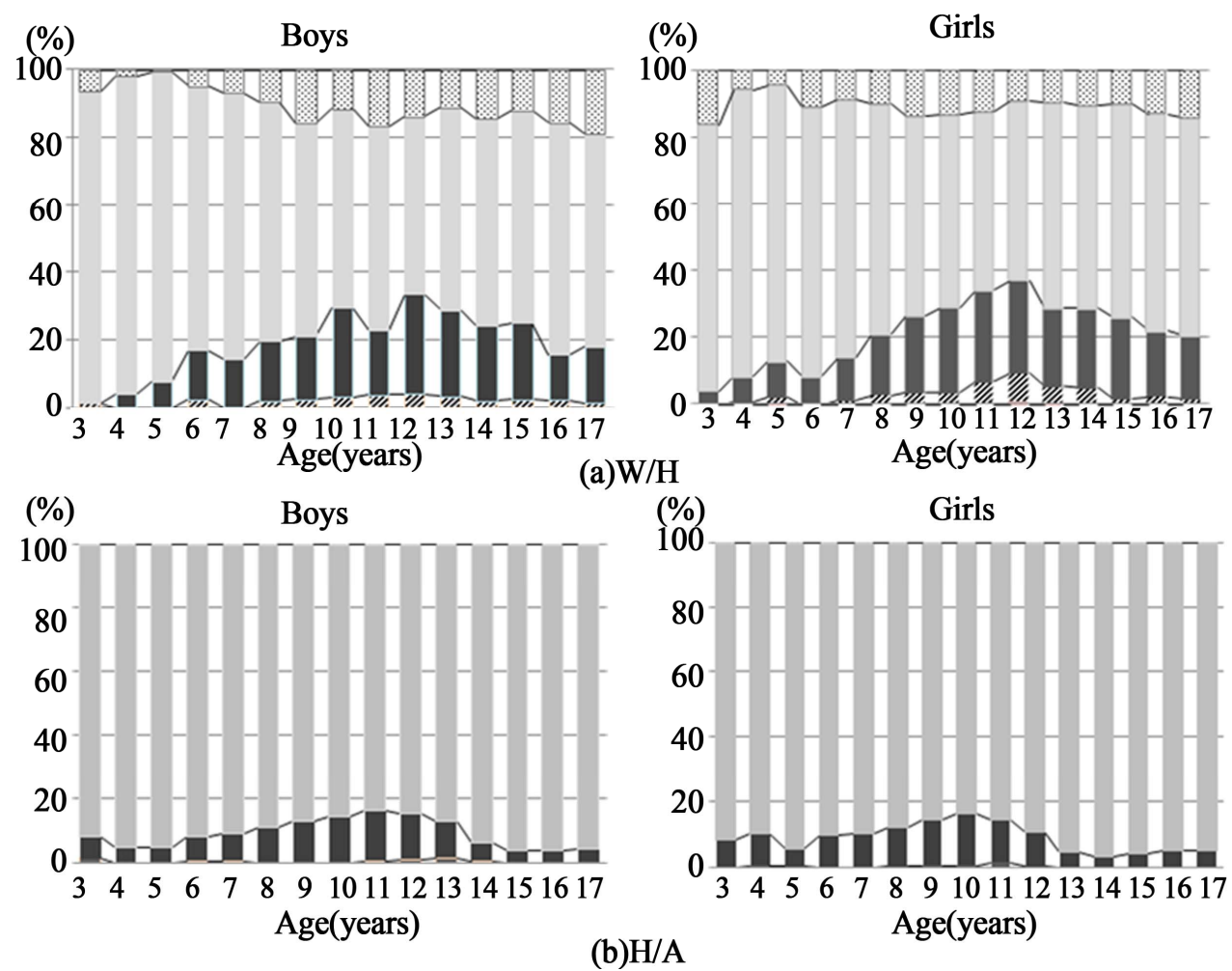

Figure 1. Changes in the prevalence of under-nutrition and overweight in $\mathrm{W} / \mathrm{H}$ and $\mathrm{H} / \mathrm{A}$ scores with age (a) W/H (weight-for-height) in boys and girls and (b) H/A (height-for-age) in boys and girls

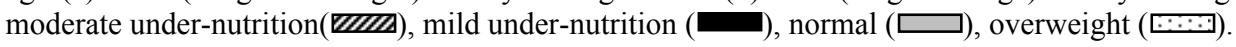

accurately perform a nutritional assessment of hospitalized children. Moreover, a recent global increase in both obesity and thinness urges us to evaluate the nutriational status in a general child population [15-17]. Based on this notion, we attempted to examine the nutritional status by using Waterlow classification in more than 7000 healthy children.

Since Waterlow classification is expressed as a percentage, it is easy for physicians to understand the results of the assessment if they become familiar with the criteria for the diagnosis of malnutrition. In addition, the measurement of both $\mathrm{H} / \mathrm{A}$ and $\mathrm{W} / \mathrm{H}$ can differentiate acute and chronic malnutrition. A disadvantage of Waterlow classification is that the collection of reference data of the standard height for the same age and sex, and standard or ideal weight for the same age and height and sex are essential. The WHO growth chart is widely used for such reference data [18]. Considering the difference of standard height and weight among children in various countries, it is desirable for each country to use their own standard for evaluating Waterlow scores. Furthermore, W/H can be underestimated, when the sample's height deviates from the median of the reference population [5]. Therefore, additional measures such as body mass index 
(BMI) z-scores [19,20] and mid-arm circumference [21] should be combined for more accurate evaluation of the nutritional assessment.

There are two major findings in the present study. First, we have demonstrated that the prevalence of under-nutrition (severe + moderate + mild) in healthy children was $8.3 \%$ by $\mathrm{H} / \mathrm{A}$ and $20.1 \%$ by $\mathrm{W} / \mathrm{H}$. On the other hand, the prevalence of overweight was $10.8 \%$ by $\mathrm{W} / \mathrm{H}$. However, the overlapping rate of under-nutrition in H/A and $\mathrm{W} / \mathrm{H}$ was approximately only $1.6 \%$. This fact may indicate, as presented initially by Waterlow et al. [11], the nature of these two types of under-nutrition is distinct. Second, from the point of age, both measures had their peaks of the number of subjects at risk of under-nutrition around 10 - 12 years old. In children, height and weight increases linearly with age during early childhood before the take-off of the pubertal growth spurt [22]. The imbalance between height and weight at puberty may cause an apparent increase of particularly under-nutrition as judged by $\mathrm{W} / \mathrm{H}$.

Our present study has several limitations. First, approximately half of the school principals did not agree to disclose student data, which may be a bias in the sampling. Second, this study was restricted to Nara city. Of note is that Nara is a provincial town with a population of 360,000 located in the middle region of Japan. The prevalence of child obesity has been shown to vary among different areas in Japan [23]. Therefore, to generalize our findings, additional studies to examine children in other areas should be performed. Finally, although the main purpose of the present study was to establish the reference values in healthy children by Waterlow classification, other nutritional assessments should be combined to make the diagnosis of malnutrition more accurately. Waterlow presented the criteria necessary for establishing a reference value. These include 1) a wellnourished population, 2) sample numbers of at least 200 individuals in each age and sex, 3) a cross-sectional study, and 4) clear and reproducible sampling procedures [11]. We believe the present study fulfills most of these criteria. Therefore, in spite of the limitations described above, the results in the present study are robust enough for general use. When physicians want to attempt nutritional assessment in hospitalized children, our data may indicate the importance of including the age factor for accurate evaluation.

\section{REFERENCES}

[1] Hendricks, K.M., Duggan, C., Gallagher, L., Carlin, A.C., Richardson, D.S., Collier, S.B., Simpson, W. and Lo, C. (1995) Malnutrition in hospitalized patients. Current prevalence. Archives of Pediatrics \& Adolescent Medicine, 149, 1118-1122.

doi:10.1001/archpedi.1995.02170230072010
[2] Pawellek, I., Dokoupil, K. and Koletzko, B. (2008) Prevalence of malnutrition in paediatric hospital patients. Clinical Nutrition, 27, 72-76. doi:10.1016/j.clnu.2007.11.001

[3] Aurangzeb, B., Whitten, K.E., Harrison, B., Mitchell, M., Kepreotes, H., Sidler, M., Lemberg, D.A. and Day, A.S. (2012) Prevalence of malnutrition and risk of under-nutrition in hospitalized children. Clinical Nutrition, 31, 3540. doi:10.1016/j.clnu.2011.08.011

[4] Den Broeder, E., Lippens, R.J., Van’t Hot, M.A., Tolboom, J.J., Van Starveren, W.A., Hofman, Z. and Sengers, R.C. (1998) Effects of naso-gastric tube feeding on the nutritional status of children with cancer. European Journal of Clinical Nutrition, 52, 494-500. doi:10.1038/sj.ejen.1600589

[5] Hirche, T.O., Hirche, H., Jungblut, S., Stern, M., Wagner, T.O. and Wiedemann, B. (2009) Statistical limitations of percent ideal body weight as measure for nutritional failure in patients with cystic fibrosis. Journal of Cystic Fibrosis, 8, 238-244. doi:10.1016/j.jef.2009.03.004

[6] Marcarenhas, M.R., Zemel, B. and Stallings, V.A. (1998) Nutritional assessment in pediatrics. Nutrition, 14, 105115. doi:10.1016/S0899-9007(97)00226-8

[7] McMahon, K. and Brown, J.K. (2000) Nutritional screening and assessment. Seminars in Oncology Nursing, 16, 106-112. doi:10.1053/on.2000.5549

[8] Pencharz, P.B. (2008) Assessment of protein nutritional status in children. Pediatric Blood Cancer, 50, 445-446. doi: $10.1002 / \mathrm{pbc} .21415$

[9] Gomez, F., Galvan, R.R., Frenk, S., Munoz, J.C., Chavez, R. and Vazquez, J. (1956) Mortality in second and third degree malnutrition. Journal of Tropical Pediatrics, 2, 1271-1274.

[10] Waterlow, J.C. (1972) Classification and definition of protein-calorie malnutrition. British Medical Journal, 3, 566-569. doi:10.1136/bmj.3.5826.566

[11] Waterlow, J.C., Buzina, R., Keller, W., Lane, J.M., Nichaman, M.Z. and Tannner, J.M. (1977) The presentation and use of height and weight data for comparing the nutritional status of groups of children under the age of 10 years. Bulletin of the World Health Organization, 55, 489-498.

[12] Reilly, H.M., Martineau, J.K., Moran, A. and Kennedy, H. (1995) Nutritional screening: Evaluation and implementation of a simple nutritional risk score. Clinical Nutrition, 14, 269-273.doi:10.1016/S0261-5614(95)80063-8

[13] The Ministry of Education, Culture, Sports, Science and Technology, Japan (2001) Reports of health statistics in school children.

http://e-stat.go.jp/SG1/estat/List.do?bid $=000001016209 \&$ cycode $=0$

[14] The Ministry of Health, Labour and Welfare, Japan (2001) Reports of growth investigation in infants and toddlers. http://www.mhlw.go.jp/houdou/0110/h1024-4.html

[15] Caballero, B. (2005) A nutrition paradox-Underweight and obesity in developing countries. New England Journal of Medicine, 352, 1514-1516.

doi:10.1056/NEJMp048310 
[16] Poskitt, E.M. (2009) Countries in transition: Underweight to obesity non-stop? Annals of Tropical Paediatrics, 29, 1-11. doi:10.1179/146532809X401971

[17] Ge, S., Kubota, M., Nagai, A., Mamemoto, K. and Kojima, C. (2011) Retrospective individual tracking of body mass index in obese and thin adolescents back to childhood. Asia Pacific Journal of Clinical Nutrition, 20, 432-437.

[18] WHO (2011) Child growth standards and the identification of severe acute malnutrition in infants and children. http://www.who.int/nutrition/publications/severemalnutrit ion/9789241598163/en /index.html

[19] Dibley, M.J., Goldsby, J.B., Staehling, N.W. and Trowbridge, F.L. (1987) Development of normalized curves for thinternational growth reference: historical and technical considerations. American Journal of Clinical Nutrition, 46, 736-748.

[20] Isanaka, S., Villamor, E., Shepherd, S. and Grais, R.F.
(2009) Assessing the impact of the introduction of the World Health Organization growth standards and weightfor-height z-score criterion on the response to treatment of severe acute malnutrition in children: Secondary data analysis. Pediatrics, 123, 54-59. doi:10.1542/peds.2008-1375

[21] Sen, J., Mondal, N. and Dey, S. (2011) Assessment of the nutritional status of children aged $5-12$ years using upper arm composition. Annals of Human Biology, 38, 752759. doi: $10.3109 / 03014460.2011 .610358$

[22] Taranger, J. and Hagg, U. (1990) The timing and duration of adolescent growth. Acta Odontologica Scandinavica, 38, 57-67. doi:10.3109/00016358008997719

[23] The Ministry of Eduction, Culture, Sports, Science and Technology, Japan (2011) Reports of health statistics in school children.

http://www.mext.go.jp/b menu/toukei/chousa05/hoken/1 268826.htm 